\title{
LEFT PRIME WEAKLY REGULAR NEAR-RINGS
}

\author{
P. DHEENA AND D. SIVAKUMAR
}

\begin{abstract}
In this paper we introduce the notion of left prime weakly regular, left prime weakly $\pi$-regular and left prime pseudo $\pi$-regular near-rings. We also introduce the concept of strong left prime weakly regular near-rings. We have obtained conditions for a near-ring $N$ to be left prime pseudo $\pi$-regular. We have also obtained conditions for a near-ring $N$ to be strong left prime pseudo $\pi$-regular. Finally we have answered the open question given in [1].
\end{abstract}

\section{Introduction}

Throughout this paper $N$ stands for a zero-symmetric near-ring. Let $P_{0}(N)$ denote the prime radical and $N(N)$ denote the set of nilpotent elements of $N$. From [2] an ideal $I$ of $N$ is a 2-primal ideal of $N$ if $P_{0}(N / I)=N(N / I)$. If $I$ is the zero ideal of $N$, then $N$ is a 2-primal near-ring (i.e. $P_{0}(N)=N(N)$ ). A near-ring $N$ is said to be reduced if $N(N)=0$.

Recall from [5] that an ideal $P$ is called a minimal prime ideal of an ideal $I$ if $P$ is minimal in the set of all prime ideals containing $I$. An ideal $I$ of $N$ is a completely prime ideal (completely semiprime ideal) if for $a, b \in N, a b \in I$ implies $a \in I$ or $b \in I\left(a^{2} \in I\right.$ implies $a \in I)$.

$N$ is said to fulfill the insertion of factors property (IFP) provided that for all $a$, $b, x \in N, a b=0$ implies $a x b=0$. Also for $X \subseteq N,(0: X)$ and $\langle x\rangle$ denote the left annihilator of $X$ and the ideal of $N$ generated by $x$ respectively.

Birkenmeier and Groenewald [1] introduced left weakly regularity in near-rings. In this paper we introduce the concept of left prime weakly regularity in near-rings.

Definition 1. (i) A near-ring $N$ is said to be left (right) prime weakly regular if for a given $x \in N$ there exists a minimal prime ideal $P$ of $\langle x\rangle$ such that $x \in P x(x \in x P)$.

(ii) $N$ is said to be left (right) prime weakly $\pi$-regular if for a given $x \in N$ there exists a natural number $n=n(x)$ and a minimal prime ideal $P$ of $\left\langle x^{n}\right\rangle$ such that $x^{n} \in P x^{n}$ $\left(x^{n} \in x^{n} P\right)$.

(iii) $N$ is said to be left (right) prime pseudo $\pi$-regular if for a given $x \in N$ there exists a natural number $n=n(x)$ and a minimal prime ideal $P$ of $\langle x\rangle$ such that $x^{n} \in P x^{n}$ $\left(x^{n} \in x^{n} P\right)$.

Following G. F. Birkenmeier and N. J. Groenewald [1], $N$ is said to be left weakly regular if $x \in\langle x\rangle x$ for all $x \in N, N$ is said to be left weakly $\pi$-regular if $x^{n} \in\left\langle x^{n}\right\rangle x^{n}$ for Received March 17, 2004; revised June 15, 2004. 
all $x \in N$ and for a natural number $n=n(x)$ and $N$ is said to be left pseudo $\pi$-regular if $x^{n} \in\langle x\rangle x^{n}$ for all $x \in N$ and for a natural number $n=n(x)$.

If $N$ is left weakly regular then clearly $N$ is left prime weakly regular. But the converse is not true as the following example shows.

Example 1. Let $N=\left[\begin{array}{ll}F & F \\ O & F\end{array}\right]$ where $F=\{0,1\}$ is the field under addition modulo 2 and multiplication modulo 2 . Then $N$ is not left weakly regular, since if $x=\left[\begin{array}{ll}0 & 1 \\ 0 & 0\end{array}\right]$, then $x \notin\langle x\rangle x=\left[\begin{array}{ll}0 & 0 \\ 0 & 0\end{array}\right]$. But $N$ is left prime weakly regular.

Lemma 2. Let $N$ be reduced. Then $N$ is left prime weakly regular if and only if $N$ is left prime pseudo $\pi$-regular.

Proof. Assume that $N$ is left prime pseudo $\pi$-regular. Let $x \in N$. Then there exists a minimal prime ideal $P$ of $\langle x\rangle$ and a natural number $n$ such that $x^{n} \in P x^{n}$. So there exists an element $a \in P$ such that $x^{n}=a x^{n}$. If $n=1$, then $x=a x \in P x$ and therefore $N$ is left prime weakly regular. For $n>1,(x-a x) x^{n-1}=x^{n}-a x^{n}=x^{n}-x^{n}=0$. Since $N$ is reduced, $N$ has the IFP. So $((x-a x) x)^{n-1}=0$ and hence $(x-a x) x=0$. Thus $x(x-a x)=0$. Since $(x-a x)^{2}=(x-a x)(x-a x)=x(x-a x)-a x(x-a x)=0$, $x-a x=0$. Thus $x=a x \in P x$. Hence $N$ is left prime weakly regular. Clearly if $N$ is left prime weakly regular then $N$ is left prime pseudo $\pi$-regular.

Theorem 3. Let $N$ be an IFP near-ring with left unity e. Then the following are equivalent:

(i) $N$ is left prime pseudo $\pi$-regular.

(ii) $N=\left(0: a^{k}\right)+P$, where $P$ is a minimal prime ideal of $\langle a\rangle$ and for some positive integer $k$.

Proof. (i) $\Rightarrow($ ii). Assume that $N$ is left prime pseudo $\pi$-regular. Let $a \in N$. Then there exists a minimal prime ideal $P$ of $\langle a\rangle$ and a positive integer $k$ such that $a^{k} \in P a^{k}$. So there exists $s \in P$ such that $a^{k}=s a^{k}$. Thus $(e-s) \in\left(0: a^{k}\right)$. Then for any $n \in N$, we have $n=(e-s+s) n=(e-s) n+s n$. Since $\left(0: a^{k}\right)$ is an ideal of $N,(e-s) n \in\left(0: a^{k}\right)$. Hence $N=\left(0: a^{k}\right)+P$.

(ii) $\Rightarrow$ (i) Let $N=\left(0: a^{k}\right)+P$ for some positive integer $k$. Then there exists $r \in\left(0: a^{k}\right)$ and $s \in P$ such that $e=r+s$. Thus $a^{k}=r a^{k}+s a^{k}=s a^{k} \in P a^{k}$. Hence $N$ is left prime pseudo $\pi$-regular.

Lemma 4. If $I=(0: a)$ is a 2-primal ideal and if $\bar{k} \bar{a} \in N(\bar{N})$ where $\bar{N}=N / I$, then $k a^{2} \in N(N)$.

Proof. Let $\bar{k} \bar{a} \in N(\bar{N})$. Since $(0: a)$ is a 2-primal ideal, $N(\bar{N})$ is an ideal. Thus $\bar{k} \bar{a}^{2} \in$ $N(\bar{N})$. So there exists a positive integer $j$, such that $\left(\bar{k} \bar{a}^{2}\right)^{j}=\overline{0}$. Thus $\left(\bar{k} \bar{a}^{2}\right)^{j}(\bar{k} \bar{a})=\overline{0}$ and hence $\left(k a^{2}\right)^{j+1}=0$. Therefore $k a^{2} \in N(N)$. 
Theorem 5. Let $N$ be a near-ring with left unity e such that every completely prime ideal is maximal. If $a \in N$ is such that $(0: a)$ is a 2-primal ideal of $N$, then there exists $s \in\langle a\rangle$ such that $a^{2}=x+s a^{2}$ where $s \in\langle a\rangle$ and $x \in N(N)$.

Proof. Let $0 \neq a \in N$ be such that $(0: a)$ is 2 -primal. Let $\bar{N}=N /(0: a)$. Since every completely prime ideal of $N$ is maximal, every completely prime ideal of $\bar{N}$ is also maximal. Let $M$ be the multiplicative semigroup generated by all elements of the form $\bar{a}-\bar{x} \bar{a}$, where $x \in\langle a\rangle$. Now we claim $P_{0}(\bar{N}) \cap M \neq \varnothing$.

Suppose $P_{0}(\bar{N}) \cap M=\varnothing$. Let $S=\{I / I$ is completely semiprime ideal with $I \cap M=$ $\varnothing\}$. Then $S$ is nonempty. Using Zorn's Lemma, $S$ has a maximal element, say $P$. Then $P$ is completely prime ideal of $N$ with $P \cap M=\varnothing$. Now $\langle\bar{a}\rangle \subseteq \bar{P}$ or there exists $\bar{\alpha} \in\langle\bar{a}\rangle$ such that $\bar{\alpha} \notin \bar{P}$. If $\langle\bar{a}\rangle \subseteq \bar{P}$, then $\bar{a}-\bar{x} \bar{a} \in \bar{P} \cap M \neq \varnothing$ for any $\bar{x} \in \bar{N}$, which is a contradiciton. So assume that there exists $\bar{\alpha} \in\langle\bar{a}\rangle$ such that $\bar{\alpha} \notin \bar{P}$. Since $\bar{P}$ is maximal, we have $\bar{P}+\langle\bar{\alpha}\rangle=\bar{N}$. Thus $\bar{e}=\bar{p}+\bar{t}$ for some $\bar{p} \in \bar{P}$ and $\bar{t} \in\langle\bar{\alpha}\rangle \subseteq\langle\bar{a}\rangle$. Then $\bar{a}-\bar{t} \bar{a}=(\bar{e}-\bar{t}) \bar{a}=\bar{p} \bar{a} \in \bar{P} \cap M \neq \varnothing$, which is again a contradiction. Therefore $P_{0}(\bar{N}) \cap M \neq \varnothing$. So we have $\left(\bar{a}-\bar{t}_{1} \bar{a}\right)\left(\bar{a}-\bar{t}_{2} \bar{a}\right) \cdots\left(\bar{a}-\bar{t}_{n} \bar{a}\right) \in P_{0}(\bar{N})$ for some $\bar{t}_{i} \in\langle\bar{a}\rangle$. Since $(0: a)$ is 2-primal, $P_{0}(\bar{N})=N(\bar{N})$. Thus $N(\bar{N})$ is completely semiprime ideal. So there exists $\bar{s} \in\langle\bar{a}\rangle$ such that $(\bar{e}-\bar{s}) \bar{a}^{n} \in N(\bar{N})$. Then $((\bar{e}-\bar{s}) \bar{a})^{n} \in N(\bar{N})$ and hence $(\bar{e}-\bar{s}) \bar{a}=\bar{k} \bar{a} \in N(\bar{N})$ where $\bar{k}=\bar{e}-\bar{s}$. Thus $(\bar{e}-\bar{s}-\bar{k}) \bar{a}=\overline{0}$ and hence $(e-s-k) a^{2}=0$. Therefore $a^{2}=k a^{2}+s a^{2}$. Since $\bar{k} \bar{a} \in N(\bar{N}), k a^{2} \in N(N)$ by Lemma 4. Hence $a^{2}=x+s a^{2}$ where $s \in\langle a\rangle$ and $x=k a^{2} \in N(N)$.

Corollary 6. ([1], Proposition 3.2 [i]) Let $N$ be an IFP right near-ring with left unity $e$ such that every completely prime ideal is maximal. If $a \in N$ such that $(0: a)$ is a 2-primal ideal of $N$, then there exists $s \in\langle a\rangle$ such that $a^{3}=s a^{3}+x$, where $x \in N(N)$.

Definition 7. (i) A near-ring $N$ is said to be strong left prime weakly regular if for a given $x \in N, x \in P x$ for every prime ideal $P$ of $\langle x\rangle$.

(ii) $N$ is said to be strong left prime weakly $\pi$-regular if for a given $x \in N$, there exists a positive integer $n$ such that $x^{n} \in P x^{n}$ for every prime ideal $P$ of $\left\langle x^{n}\right\rangle$.

(iii) $N$ is said to be strong left prime pseudo $\pi$-regular if for a given $x \in N$ there exists a positive integer $n$ such that $x^{n} \in P x^{n}$ for every prime ideal $P$ of $\langle x\rangle$.

If $N$ is left weakly regular then clearly $N$ is strong left prime weakly regular. For example, $\left(Z_{6}, \oplus, \odot\right)$ is strong left prime weakly regular, where $\oplus$ and $\odot$ are the addition modulo 6 and multiplication modulo 6 respectively.

If $N$ is strong left prime weakly regular, then $N$ is left prime weakly regular. But the converse is not true as the following example shows.

Example 2. The near-ring $N$ in Example (1) is left prime weakly regular but not strong left prime weakly regular, since for $P=\left\{\left[\begin{array}{ll}0 & 0 \\ 0 & 0\end{array}\right],\left[\begin{array}{ll}0 & 1 \\ 0 & 0\end{array}\right],\left[\begin{array}{ll}0 & 1 \\ 0 & 1\end{array}\right],\left[\begin{array}{ll}0 & 0 \\ 0 & 1\end{array}\right]\right\}$ and for $x=\left[\begin{array}{ll}0 & 1 \\ 0 & 0\end{array}\right], x \notin P x=\left[\begin{array}{ll}0 & 0 \\ 0 & 0\end{array}\right]$. 
Theorem 8. If $N$ is 2-primal and $N$ is strong left prime pseudo $\pi$-regular, then every prime ideal $P$ of $N$ is completely prime.

Proof. Let $P$ be any prime ideal of $N$. Since $N$ is 2-primal, $P_{0}(N)=N(N)$ and hence $P_{0}(N)$ is completely semiprime. So by Proposition 2.1 of [1] there exists a prime ideal $X$ of $N$ which is minimal among prime ideals and $X \subset P$ is completely prime.

Suppose $Q$ is any prime ideal such that $X \subset Q$. Let $a \in Q$ be such that $a \notin X$. Since $N$ is strong left prime weakly regular, $a^{k} \in Q a^{k}$ for some positive integer $k$. Thus $a^{k}=q a^{k}$ for some $q \in Q$. So for $n \in N, n a^{k}=n q a^{k}$. Hence $(n-n q) a^{k}=0 \in X$. Since $X$ is completely prime and $a \notin X$, we have $n-n q \in X \subset Q$. Thus $n \in Q$, which implies $Q=N$. Hence $X=P$ and $P$ is completely prime.

Definition 9. A near-ring $N$ is said to satisfy $P I$ condiiton if for any ideal $I$ of $N$ and for any $x \in I$, there exists a prime ideal $P$ of $N$ such that $\langle x\rangle \subseteq P \subseteq I$.

Theorem 10. If $N$ is 2-primal with PI condition and strong left prime pseudo $\pi$-regular, then every prime ideal of $N$ is maximal.

Proof. Let $P$ be any prime ideal of $N$. Since $N$ is 2-primal, $P_{0}(N)$ is completely semiprime. So by Propositon 2.1 of (i), there exists a completely prime ideal $X$ of $N$ such that $X \subset P$. Let $I$ be any ideal such that $X \subset I$ and let $a \in I$. Since $N$ satisfies $P I$ condition, there exists a prime ideal $Q$ of $N$ such that $\langle a\rangle \subseteq Q \subseteq I$. Since $N$ is strong left prime psuedo $\pi$-regular, we have $a^{k} \in Q a^{k}$ for some positive integer $k$. As in the case of Theorem 8 , we get $Q=N$. Therefore $I=N$. Thus $X=P$ and hence $P$ is maximal.

Following [1], $N$ satisfies the CZ1 condition if for any $x, y \in N$ and positive integer $k$ such that $(x y)^{k}=0$, then there exists a positive integer $m$ such that $x^{m} y^{m}=0$.

Theorem 11. Let $N$ be a near-ring with left unity e which satisfies the CZ1 and PI condition. Suppose that $(0: a)$ is a 2-primal ideal for all $a \in N$. Then the following are equivalent:

(i) $N$ is left pseudo $\pi$-regular.

(ii) $N$ is strong left prime pseudo $\pi$-regular.

(iii) Every prime ideal is maximal.

(iv) Every completely prime ideal is maximal.

Proof. (i) $\Rightarrow$ (ii) Since $N$ is left pseudo $\pi$-regular, for every $x \in N$ there exists a natural number $n=n(x)$ such that $x^{n} \in\langle x\rangle x^{n}$. Therefore $x^{n} \in P x^{n}$ for every prime ideal $P$ of $\langle x\rangle$. Hence $N$ is strong left prime pseudo $\pi$-regular.

(ii) $\Rightarrow$ (iii) Since $N$ is strong left prime pseudo $\pi$-regular, by Theorem 10 every prime ideal of $N$ is maximal.

(iii) $\Rightarrow$ (iv) Proof is immediate.

(iv) $\Rightarrow$ (i) Let $a \in N$. Since every completely prime ideal is maximal, by Theorem 5 there exists $s \in\langle a\rangle$ such that $a^{2}=x+s a^{2}$ for some $x \in N(N)$. Thus $\left((e-s) a^{2}\right)^{k}=0$ for some positive integer $k$. Since $N$ satsifies CZ1 condition, there exists a positive 
integer $m$ such that $(e-s)^{m} a^{2 m}=0$. Thus $(e-\bar{s}) a^{2 m}=0$ for some $\bar{s} \in\langle a\rangle$. Hence $a^{2 m}=\bar{s} a^{2 m} \in\langle a\rangle a^{2 m}$.

Birkenmeier and Groenewald [1] have raised the following question: Is a reduced left weakly regular near-ring (with unity) also a right weakly regular? We have answered affirmatively.

Theorem 12. A reduced left weakly regular near-ring is also a right weakly regular.

Proof. Let $N$ be a left weakly regular near-ring. Let $x \in N$. Then $x \in\langle x\rangle x$. So there exists $a \in\langle x\rangle$ such that $x=a x$. Thus $(x-x a) x=x^{2}-x a x=0$. Since $N$ is reduced, we have $x(x-x a)=0$ and hence $x a(x-x a)=0$. Then $(x-x a)^{2}=(x-x a)(x-x a)=$ $x(x-x a)-x a(x-x a)=0$ and hence $x-x a=0$. Thus $x=x a \in x\langle x\rangle$. Therefore $N$ is right weakly regular.

\section{References}

[1] G. F. Birkenmeier and N. J. Groenewald, Near-rings in which each prime factor is simple, Mathematics Pannonica 10(1999), 257-269.

[2] G. F. Birkenmeier, H. Heatherly and E. Lee, Prime ideals and prime radicals in near-rings, Mh. Math 117(1994), 179-197.

[3] C. Y. Hong and T. K. Kwak, On minimal strongly prime ideals, Comm. in Algebra 28 (2000), 4867-4878.

[4] N. K. Kim and T. K. Kwak, Minimal prime ideals in 2-primal rings, Math. Japonica 50 (1999), 415-420.

[5] G. Pilz, Near-Rings, North-Holland, Amsterdam, 1983.

Department of Mathematics, Annmalai University, India.

Department of Mathematics, D.D.E., Annamalai University, India. 